\title{
TOULMIN: RAZONAMIENTO, SENTIDO COMÚN Y DERROTABILIDAD ${ }^{1}$
}

\author{
Claudio Fuentes Bravo* \\ claudio.fuentes@udcp.cl \\ Cristián Santibáñez Yãnez** \\ cristian.santibanez@udcp.cl
}

RESUMO Primeiramente, oferecemos uma apresentação teórica da representação do pensamento prático, começando pela distinção entre silogismo dialético e silogismo demonstrativo. Fazemos referência à crítica de Toulmin contra o dedutivismo dominante de seu tempo. Em seguida, fornecemos argumentos para apoiar a relevância heurística do modelo de Toulmin para entender a discussão sobre a inclusão da lógica padrão na representação do pensamento comum. Afirmamos que o projeto analítico toulmaniano permite entender, com clareza metódica, a derrotabilidade dos argumentos do senso comum por meio da investigação da noção modal de probabilidade na linguagem comum.

Palavras-chave Esquemas argumentativos, derrotabilidade, garantia inferencial, pensamento prático, modelo analítico de Toulmin.

1 Trabajo producido en el marco del Proyecto Fondecyt 1130584, "Valor, función y complejidad argumentativa en estudiantes universitarios chilenos: el caso en las regiones de Coquimbo y Metropolitana".

* Professor do Centro de Estudios de la Argumentación y el Razonamiento, Universidad Diego Portales, Chile.

** Professor do Centro de Estudios de la Argumentación y el Razonamiento, Universidad Diego Portales, Chile. Artigo recebido em 14/06/2013 e aprovado em 30/10/2013. 
ABSTRACT First, weoffer theoretical resemblance of the representation of practical reasoning, beginning with the distinction between demonstrative and dialectical syllogism. We make reference to the Toulmin's critique against to the dominant deductivism in his time. Then we give arguments to sustain the heuristic relevance of Toulmin's model to understand the discussion about the inclusion of default logics in the representation of ordinary reasoning. We assert that the Toulminian analytic design allows to understand, with methodical clarity, the defeasibility of common sense arguments by means of the investigation of the modal notion of probability in ordinary language.

Keywords Argumentative schemes, defeasibility, inferential warrant, practical reasoning, Toulmin's analytic model.

\section{Introducción}

Durante siglos, la lógica aristotélica, representada fundamentalmente por el formalismo deductivo que hoy conocemos como silogismo demostrativo, se constituyó en el modelo excluyente de la racionalidad científica. Como bien lo recuerda Evans (1999, p. 44) introduciendo "Tópicos" de Aristóteles: "Un silogismo es demostrativo o científico cuando sus premisas son intrínsecamente verdaderas, ya porque son conocidas por sí mismas con una evidencia absoluta (axiomas), ya porque son deducidas a partir de axiomas (teoremas ya demostrados)."

Sin embargo, en "Tópicos" Aristóteles ofrece una definición de silogismo menos restrictiva que corresponde a la de silogismo dialéctico. Dice allí: "Un razonamiento silogístico (dialéctico) es una fórmula de argumentación en la cual, habiéndose planteado ciertas cosas, una cosa distinta de las que fueron planteadas se sigue necesariamente, por la virtud misma de lo que fue planteado" (Aristóteles, "Tópicos", 100a, 25-27).Cuando se refiere a "ciertas cosas que son planteadas", Aristóteles está pensando en un tipo especial de premisas que llama endójicas (concepto que Brunschwig (1967) tradujo acertadamente -a nuestro juicio- como ideas admitidas). Otra afirmación de Aristóteles en "Tópicos", que nos parece relevante destacar en este contexto, es que tales ideas se dicen admitidas en relación con el sujeto que las sostiene, a la cantidad y a las circunstancias en que lo hace. Así, podemos decir que son admitidas "[...] por todos los hombres, o por casi todos, o por aquellos que representan una opinión esclarecida; $\mathrm{y}$, entre estos últimos, bien por todos, o 
bien por casi todos, o bien por los más conocidos" (Aristóteles, "Tópicos", 100b, 26-101a1).

El concepto de premisa aristotélico se puede vincular a la noción más moderna, y de cierta manera más amplia, de garante inferencial. Este último concepto se puede dividir, a su vez, en dos categorías: garante inferencial no deductivo y garante inferencial deductivo. Una distinción que Govier (1987, p. 37) ha llamado the great divide. Como se sabe, subyace a esta división una especie de teoría del déficit, según la cual los argumentos deductivos determinarían un estándar (un conjunto de propiedades determinadas) que no cumplirían los inductivos. Es decir, los garantes inductivos se pueden definir como garantes deficitarios, desde el punto de vista omnisciente del sujeto racional de la lógica clásica. No obstante, los garantes deficitarios serían útiles para representar propósitos epistémicos para los que los garantes deductivos parecen demasiado restrictivos. Govier (1987) sugiere además que una teoría que afirme la existencia de sólo dos clases de argumentos, a saber, deductivos e inductivos, puede ser denominada teoría positivista del argumento.

La noción de garante inferencial nos informa de la posibilidad de establecer una comparación entre grados de fuerza con los que las premisas apoyan a su conclusión en los dos tipos de silogismos descritos. Asumiendo además que estamos en un contexto dualista, en el caso del silogismo dialéctico, la fuerza con la que apoyan las premisas endójicas a su conclusión es menor a la fuerza con la que apoyan las premisas categóricas su conclusión en un silogismo demostrativo.

Los garantes no deductivos (premisas endójicas) evocan inevitablemente entonces la noción de garantía en el modelo analítico de Stephen Toulmin. La conocida querella contra del deductivismo que Stephen Toulmin presenta en "Los usos de la argumentación" ([1958] 2007, p. 13, en adelante LUA), será un punto de inflexión para las investigaciones en torno al razonamiento práctico, lo que relegará a un segundo plano los objetivos prioritarios de las investigaciones de corriente principal, como lo era la validez y consistencia del razonamiento (Velasco, 2007, p. 362).

Para Gabbay y Woods (2002) la tendencia rupturista que apuntamos en el párrafo anterior, que a nuestro juicio tiene uno de sus puntos de partida en el trabajo de Toulmin (1958), inaugura el desarrollo de un nuevo paradigma inferencial, el que debe tomar en cuenta el contexto complejo de la información que procesa todo agente cognitivo.

Lo que deja de funcionar, si suscribimos la crítica de Toulmin, es una teoría del déficit, que como hemos propuesto subyace a toda teoría dualista. Esto se debe poner en perspectiva. Recién a mediados del siglo XX, con la 
pérdida de influencia del paradigma positivista en filosofía, se brindó nueva atención a la relación entre percepción sensorial y mundo externo. Los hechos epistemológicos paradigmáticos son expresados usando sentencias tales como las de la lista de Goldman (1979, pp. 1-2): "Justificado, garantizado, tiene (buen) fundamento, tiene razón (para creer), sabe que, observa que, aprende que, es probable (en un sentido epistémico o inductivo), muestra que, establece que, comprueba que". El asunto crucial sobre sentencias que usan estos términos, es que parecen más que limitarse a describir cómo son las cosas. Dicen o implican cómo se va a evaluar algo desde una perspectiva epistemológica. Los epistemólogos tradicionales toman estas sentencias epistemológicas evaluativas como proposiciones susceptibles de ser objetivamente verdaderas o falsas, por lo quese comprometen con la existencia de hechos epistemológicos.

Por su parte, Chisholm (1977) sostiene que hay principios de evidencia distintos a los principios formales de lógica deductiva e inductiva (Chisholm, 1977, p. 67). Por ejemplo, hay principios especiales sobre evidencia perceptual en el sentido de: (R) estar en un estado de parecer ver algo rojo (ser aparentemente rojizo), es evidencia para la proposición que uno realmente ve algo rojo. El apoyo evidencial descrito en $(\mathrm{R})$ es derrotable. Uno podría tener evidencia que uno realmente no ve algo que parece rojo a pesar de que las cosas parecían rojizas. La cuestión clave sobre (R) es que no es, o al menos no obviamente, un ejemplo de algún principio probabilístico deductivo o inductivo general. No se trata entonces de un principio lógico al modo de la lógica tradicional, sino de un principio inferencial derrotable, fundamental para la epistemología contemporánea, al menos a partir de "Knowledge and Justification" de Pollock (1974). Los tradicionalistas argumenten que estos principios son casos especiales de principios generales aceptables naturalmente. Por ejemplo, un podría argumentar que $(\mathrm{R})$ se sigue de algún principio general acerca de la mejor explicación, tal como lo expone Harman (1965).

Pollock desarrolló el punto de vista de Chisholm dentro de su teoría de las razones prima facie y los derrotadores de esas razones (Pollock, 1967; Pollock, 1974). Distinguió entre dos tipos de derrotadores de inferencias derrotables: los rebutting defeaters (que ponen en duda a través de una razón prima facie la negación de la conclusion original) y undercutting defeaters (que ponen en duda la relacióninferencial entre las premisas y su conclusión). De acuerdo con Pollock, una conclusión está garantizada dadas todas las evidencias de algo, si es apoyada a la larga por un argumento no derrotable cuyas premisas son extraídas de esta evidencia. 
Es fácilmente constatable en la experiencia cotidiana que el razonamiento humano implica inevitablemente el uso de estrategias de inferencia más flexibles y expresivas que las prescritas por la corrección formal de los razonamientos deductivos. A estas estrategias de inferencia más flexibles y expresivas, se les ha denominado con frecuencia razonamiento de sentido común, y podríamos caracterizarlas como un tipo de razonamiento que apela -en el mejor de los casos-, a una corrección material, es decir, a cumplir con la condición que establece que la verdad de las premisas de un razonamiento dado debe constituir, en el contexto en el que aparece, una garantía adecuada de la verdad de su conclusión.

Ahora bien, los intentos de formalización del razonamiento de sentido común, como por ejemplo los formulismos de Reiter para representar inferencias por defecto (Reiter, 1980), o los sistemas de argumentación derrotable de Prakken y Vreeswijk (2002), muestran una íntima conexión “[...] con viejos problemas ya planteados en aplicaciones científicas, (suposiciones "ceteris paribus"), deónticas (obligaciones "prima facie"), del discurso cotidiano ("condicionales contrafácticos"), etc." (Carnota, 2005, p. 144).

En suma, para comprender este vasto asunto, tengamos presente que si definimos a la lógica del razonamiento deductivo como el estudio de la relación de consecuencia deductiva, que contiene a su vez de forma distintiva la propiedad de monotonía, el rechazo de Toulmin a la inferencia deductiva implicaría cerrar la posibilidad de hacer converger un diseño de representación del razonamiento práctico con cualquier modelo de representación basado en sistemas lógicos monótonos. No obstante, Toulmin deja abierta la posibilidad de establecer una convergencia entre un diseño de representación de razonamiento, con algún sistema de lógica no monótona, como por ejemplo, las lógicas derrotables.

Para una analogía general entre los modelos de razonamiento derrotable y el modelo analítico de Toulmin, resulta interesante considerar en este punto la relevancia que ha adquirido en los sistemas de lógica derrotable el estudio de los criterios de garantía para obtener una conclusión, es decir, los criterios que usamos para determinar el grado según el cual es razonable aceptar ciertas conclusiones, teniendo en cuenta que su obtención no siempre ha sido deductiva y no puede sostenerse con absoluta certeza.

Toulmin en LUA ilustra la idea precedente al referirse al concepto de garantía como sigue:

Las garantías son de diferente clase, por lo que confieren diversos grados de fuerza a las conclusiones que justifican. Algunas garantías permiten aceptar una afirmación de manera inequívoca si se cuenta con los datos apropiados: son garantías que, 
en los casos adecuados, nos permiten matizar nuestra conclusión con el adverbio "necesariamente"; otras nos permiten dar el paso de los datos a las conclusiones, ya sea provisionalmente, ya sujetas a condiciones, excepciones o matizaciones: en estos casos hay que emplear otros términos modales, tales como "probablemente" o "presumiblemente". (Toulmin, 2007, p. 137)

Esta preocupación por las formas en que es posible garantizar una conclusión de forma no deductiva, generó un emergente campo de investigación en el ámbito de las lógicas derrotables que es conocida hoy como argumentación derrotable. Probablemente el primero que realizó un análisis lógico de las inferencias derrotables, según Alchourrón (1993), fue Hansson (1969) con su propuesta de lógica deóntica diádica y su formalización de la obligación condicional.

La argumentación derrotable ha evolucionado actualmente desde un enfoque que se ha denominado lógica retractable clásica, a partir tanto de la formalización de Billington (1993), como del enfoque epistemológico particularmente influenciado por las ideas de Pollock en una serie de publicaciones que van desde "Conocimiento y Justificación" (1974) hasta "Defeasible reasoning with variable degrees of justification" (2001) pasando por "Razonamiento derrotable" (1987), solo por nombrar algunos de sus trabajos; y ha llegado en nuestros días a concretarse en diferentes propuestas de formalización para el razonamiento de sentido común que describen fundamentos epistemológicos diversos, lo que podemos apreciar en los trabajos de Nute (1994), Prakken y Vreeswijk, 2002), Maher et al. (2001), Chesñevar, Maguitman y Loui (2000), y Paglieri y Castelfranchi (2005), entre otros.

No obstante, en relación con los objetivos acotados de este manuscrito, lo relevante de señalar aquí es que el diseño analítico de Toulmin permite constatar con claridad metódica la derrotabilidad de los argumentos de sentido común, a través de la investigación de la noción modal de probabilidad en el lenguaje corriente, lo que a su vez da paso a la comprensión de la argumentación como un proceso epistémico dinámico y multi-agente. Al respecto Paglieri y Castelfranchi (2005, p. 9) sostienen lo siguiente: “[...] la teoría de Toulmin resulta ser crucial [...], tanto para la evaluación crítica de la existencia de formalismos de cambio doxástico, como para inspirar nuevos modelos en tanto marco de referencia."

Siendo consciente de la larga, pero necesaria, introducción que hemos desarrollado, es en este marco que los siguientes desarrollos deben concebirse. La propuesta de Toulmin, a nuestro juicio, debería observarse como impulso seminal de la modelización del razonamiento práctico y no-monotónico que 
conoce en los avances contemporáneos un eco claro y revelador. En lo que sigue, discutiremos la posibilidad de construcción de modelos analíticos para el estudio del razonamiento práctico; luego, abordaremos con cierto detalle la aproximación toulminiana; para desde allí explicar por qué y cómo la noción de esquemas argumentativos se puede anclar en una ruta inaugurada, creemos, por Toulmin. En las conclusiones enfatizamos los aspectos más importantes de nuestro acercamiento al problema general de la derrotabilidad.

\section{La construcción de modelos analíticos para el análisis del razonamiento práctico}

Un contexto discursivo propicio para el razonamiento derrotable ha sido históricamente la exposición y análisis de controversias en la praxis jurídica, en donde son comunes las nociones de argumento y contra argumento, réplica y derrota (Prakken y Vreeswijk, 2002). Esto lo podemos constatar en la utilización que Ross (1930) desarrolló del concepto prima facie, esto es, la idea de un deber preponderante o condicionado, susceptible de ser derrotado o anulado en un contexto particular en donde otro deber es aplicable con mayor fuerza o pertinencia que el primero, siendo a su vez inconsistente con él. El concepto prima facie es recuperado por Pollock (1987) como marco de referencia para la representación del razonamiento derrotable. En la obra de Pollock (1995) este conceptoes especialmente relevante, ya que clasifica reglas de inferencia derrotable según los principios generales de la epistemología y el razonamiento práctico. Pollock llama a su regla de inferencia derrotable razones prima facie. Una de éstas es el principio de la percepción: Tener una percepción con contenido $\mathrm{p}$ es una razón prima facie para creer que p. Bajo la nomenclatura razones prima facie Pollock se refiere, por ejemplo, al silogismo estadístico (una versión probabilística del modus ponens derrotable) y los principios de la memoria, la inducción y la persistencia temporal (Prakken, 2005).

Pollock señala que los argumentos puedan ser atacados de dos maneras. Un argumento puede ser refutado con un argumento para la conclusión contraria (rebutting defeaters), pero también pueden ser debilitado con un argumento con una razón prima facie (undercutting defeaters) que arroje dudas sobre el argumento a partir de las ramificaciones posibles, esto es, el argumento de ataque se obtiene por la cadena de razones implicadas que debilitan al argumento en cuestión y no por una oposición directa. Intuitivamente, en los ataques de debilitamiento no se discute que la conclusión sea falsa, sino sólo que la conclusión no está suficientemente apoyada por sus premisas. 
La noción de debilitamiento permite apreciar la dependencia de campo de los estándares para la evaluación de argumentos (Toulmin, 2007), ya que cada argumento tiene sus propios debilitadores (undercutters) dependiendo del campo argumentativo respecto del cual provienen sus garantes (campos jurídico, económico, científico, artístico, etc.).

Lo crucial, desde el punto de vista analítico y metodológico, es que la modelización permitiría trabajar sobre el siguiente problema: dado un conjunto de argumentos en conflicto, es preciso determinar si algunos de estos argumentos prevalecerán. Esto se podría obtener siguiendo dos pasos (Horty, 2007, p. 3):

1. Primero, considerando las situaciones en las que establecen relaciones de prioridad entre razones, utilizando razonamiento derrotable.

2. Segundo, aplicando a razones en conflicto un tratamiento basado en contraargumentos por debilitamiento y razones excluyentes.

En línea con los resultados teóricos de la filosofía práctica, moral y jurídica, de los cuales son ejemplos el concepto de deber prima facie de Ross, y de cierto modo la definición derrotable de concepto jurídico de Hart, Toulmin (2007) asume la tarea de reemplazar el modelo deductivista de análisis de argumentos sustanciales, por un esquema procedimental análogo a las prácticas normativas sedimentadas en la jurisprudencia:

La lógica, podríamos decir, es una jurisprudencia generalizada. Los argumentos pueden compararse con las demandas judiciales. [...] Una de las tareas capitales de la jurisprudencia es caracterizar los elementos esenciales del proceso legal: los procedimientos o trámites por medio de los cuales las demandas se presentan ante la ley, se debaten y se determinan, así como las categorías en que esto se lleva a cabo. [...] nuestra finalidad es, análogamente, caracterizar lo que podría llamarse "el proceso racional", los trámites y categorías que se emplean para que las afirmaciones en general puedan ser objeto de argumentación y el acuerdo final sea posible. (Toulmin, 2007, p. 25)

Sin embargo, para Loui (1995) no podemos considerar a Ross como el precursor de los estudios de la derrotabilidad en la filosofía jurídica; ese honor debe estar reservado para Hart quien enuncia una observación fundamental para tal efecto, en "The Ascription of Responsibility and Rights" de 1949: "[...] los conceptos jurídicos son derrotables si las condiciones en virtud de las cuales decimos que una situación es clasificable como ejemplo de un concepto jurídico, son sólo normalmente, o presuntamente, suficientes" (Hart, 1949, p. 151). 
La naturaleza de los problemas tratados en LUA, según su autor, son lógicos, pero no lo son al modo en que lo serían unos problemas típicos de la disciplina lógica tradicional, sino que son lógicos en un sentido general: “[...] surgen de la preocupación por cómo se aplican los argumentos en la práctica y por las relaciones que tienen con los cánones y los métodos que se usan cuando, en la vida diaria, evaluamos la validez, la fuerza y el carácter concluyente de los argumentos" (Toulmin, 2007, p. 9).

La investigación filosófica de Toulmin se orienta a problemas lógicos si entendemos por problema, básicamente, la necesidad construir un modelo de evaluación del razonamiento, y por lógica en sentido general, el estudio de patrones, cánones o estructuras estables en el uso del lenguaje corriente cuando evaluamos la validez, la fuerza o la garantía que tenemos para extraer una conclusión a partir de ciertas premisas.

\section{El método analítico de Toulmin}

En el primer capítulo de LUA, Toulmin intentó describir a grandes rasgos la estructura que adoptan los argumentos en la práctica, así como las características más destacadas de las categorías empleadas en la evaluación real de esos argumentos. El autor planteó allí la posibilidad de distinguir aquellos elementos invariables en los argumentos procedentes de diversos campos, de aquellos otros dependientes del campo desde donde surgen. El procedimiento elegido por el autor consiste en tomar un término modal, como por ejemplo, posible o probable, y ver qué permanece idéntico y qué cambia cuando consideramos el modo característico en que se emplea, primero en un campo de argumentación determinado y luego en otros (Toulmin, 2007, p. 42).

Si seguimos la argumentación de Toulmin, la palabra imposibilidad en tanto término modal, supone en el uso cotidiano del lenguaje la siguiente idea: descartar una posibilidad relevante. No obstante, las razones implícitas para descartar una acción y la sanción que se corre el riesgo de sufrir por no obedecer la orden varían según el caso. Estas fluctuaciones son de especial interés para la propuesta de Toulmin:

Sujeto a estas condiciones queda lo que es común a todas las afirmaciones de 'imposibilidad'. Todas ellas pueden escribirse siguiendo el siguiente esquema en el que se ponen de manifiesto las implicaciones que se encierran en ellas: "Siendo P lo que es, se debe descartar cualquier cosa que suponga $\mathrm{Q}$, actuar de otro modo sería $\mathrm{R}$ y daría lugar a S". Esta forma es común a todos los ejemplos; lo que varía de caso a caso son los elementos que sustituyen a P, Q, R y S. (Toulmin, 2007, p. 51) 
El resultado en este caso para Toulmin, es la regimentación de una porción del lenguaje corriente para el caso del uso de un término modal. Es posible entonces, para él, en la práctica argumentativa, sostener una forma común invariante para un término modal mientras los elementos variables del mismo pueden ser sustituidos según el caso sin alterar el sentido del término.

En LUA su autor también se interroga por la manera en que los resultados de este tipo de análisis pueden ser relevantes para el estudio de cuestiones y problemas filosóficos, como por ejemplo, el de la probabilidad. El ejemplo a continuación pretende ilustrar el uso de la noción de probabilidad en el lenguaje corriente a través del adverbio probablemente, no obstante podemos observar en él también la derrotabilidad de las conclusiones: "Al decir "S es probablemente P" o "Haré probablemente A", evito explícitamente comprometerme sin reservas, asegurándome de este modo contra algunas de las consecuencias en caso de incumplimiento: mi enunciado queda así a "cubierto cautelar", esto es, según la definición del "Pocket Oxford Dictionary", a "salvo condicional, por estipulación, de abuso o malinterpretación” (Toulmin, 2007, p. 75).

La clave del análisis modal que nos ofrece Toulmin de los ejemplos que fundamentan su punto de vista, es la comprensión intuitiva del adverbio probablemente. El autor parte de un supuesto psicogenético del que -dicho sea de paso- no entrega un respaldo empírico. Afirma que el adverbio probablemente se adquiere con anterioridad a la noción de probabilidad. Es decir, asumimos que el uso de formas adverbiales como probablemente responden más cercanamente a procesos dialógicos de semantización que el dominio de un significado particular del sustantivo probabilidad. El adverbio probablemente, siguiendo el razonamiento del autor, se utiliza entonces en la comunicación cotidiana como una herramienta eficaz para matizar las conclusiones y aseveraciones, indicando que un enunciado tiene un carácter no del todo categórico, lo que exhibe una ganancia subjetiva muy atractiva para el hablante, esto es, no lo compromete pragmáticamente. La familia de palabras vinculadas a probabilidad como núcleo de sentido, sirven tanto para matizar las propias aseveraciones, promesas y evaluaciones, como también para indicar la fuerza que posee el apoyo que motiva esas actitudes proposicionales. Toulmin estima, en relación con la idea anterior, que depende de la calidad de los datos o pruebas a disposición del agente racional, el tipo de matización que puede incluir en sus afirmaciones (necesidad, probabilidad, plausibilidad).

Ahora bien, lo que resulta de particular interés al adaptar el esquema de Toulmin al modo tradicional silogístico, es la función del calificador, que 
curiosamente aparecería, una vez hecha la conversión al modo tradicional, en la conclusión. Este sería un rasgo relevante para el planteamiento de una analogía general entre el modelo de Toulmin y la representación de la inferencia derrotable.

Según Paglieri y Castelfranchi (2005, p. 5) lo que llamamos modelo de Toulmin puede resumirse de la siguiente manera:

El diseño de Toulmin distingue y analiza seis características de un argumento: los datos, la pretensión, la garantía, el apoyo, el cualificador y la refutación. Los datos son los hechos (por ejemplo, "John amaba a su mujer"), que apoyan la pretensión del argumentador (por ejemplo, "Juan no la asesinó"), mientras que la garantía asegura la conexión entre los datos y la pretensión (por ejemplo, "la gente no asesina a los seres queridos"), sobre la base de algún apoyo (por ejemplo, evidencias estadísticas de que la gente no mata a aquellos que ama), el cualificador especifica hasta qué punto la garantía es aplicable (por ejemplo, "por lo general"), y la refutación describe las excepciones a la garantía (por ejemplo, "A menos que Juan haya buscada o beneficiarse del seguro de vida de su mujer").

El término hecho puede asumirse como un elemento de información que es específica a un contexto dado. Por ejemplo, considere un médico que asesora a un paciente. Los hechos son la información sobre un determinado paciente, tales como nombre, edad y la presión arterial. Esta información sólo es aplicable a ese paciente. Esto contrasta con el conocimiento, en forma de normas tal vez derrotables, con principios tales como: si un paciente tiene presión arterial alta y es de mediana edad, entonces, recetar una dieta baja en sodio. En esencia, se dice que si las condiciones requeridas (representadas por los hechos y la garantía) se cumplen, entonces hay una razón para aceptar la pretensión cualificada. Para esta configuración, podemos considerar que los hechos orientan el tipo de apoyo que debe recibir un argumento. El apoyo, o respaldo, es una especie de justificación que proporciona una explicación de por qué la pretensión (datos, garantía y conclusión) es una razón para aceptar la afirmación cualificada. Las justificaciones pueden basarse en diversos criterios, tales como la creencia, el derecho, la autoridad, la ética, la moral o la estética. La refutación capta las circunstancias que serían consideradas como excepciones para la pretensión esgrimida. Esto es, si atendemos a la información sustantiva que nos permite llegar a la conclusión, nos vemos enfrentados al potencial bloqueo del argumento, es decir, a la incorporación de cambios en la información sustantiva de los antecedentes. Estos cambios son captados en el modelo a través de la función del calificador (Q) y las condiciones de refutación $(\mathrm{R})$ : "Los calificadores o matizadores $(\mathrm{M})$ indican la fuerza conferida por la garantía en el paso adoptado, mientras que las condiciones de refutación (E) apuntan a las circunstancias en que la autoridad 
general de la garantía ha de dejarse a un lado. Por tanto, para garantizar una conclusión el argumento no solo debe proveer un conjunto determinado de datos, sino que debe dar cuenta fundamentalmente de la naturaleza y justificación del vínculo entre datos y conclusión" (Toulmin, 2007, p. 133). Toulmin insiste en esta idea:

[...] lo que hay que hacer no es ofrecer más datos [...], sino proposiciones de un tipo bastante diferente: reglas, principios, enunciados, etc., que nos permitan realizar inferencias en lugar de agregar información adicional. [...] llegados a este punto lo que se necesita, por lo tanto, son enunciados hipotéticos, de carácter general [...]. Normalmente esos enunciados hipotéticos pueden formularse de manera muy sucinta (siguiendo el esquema "si D entonces C") o [...] "Datos tales como D permiten extraer conclusiones o realizar afirmaciones tales como C"; o alternativamente: "Dados los datos D, puede asegurarse que C". (Toulmin, 2007, p. 134)

A las proposiciones definidas en la cita precedente, Toulmin las llamó garantías, y constituye el concepto central del modelo de representación de un argumento en LUA. Paglieri y Castelfranchi (2005) han llamado la atención sobre la similitud del concepto de garantía de Toulmin y el concepto que Pierce denominó principio rector de una clase de inferencias, las que el pragmatista estadounidense definió como "una proposición relacionada con el hábito de inferencia que establece que cualquier proposición $c$ que se relaciona de una manera general con cualquier proposición verdadera $p$ es verdadera" (Peirce, 1955, p. 131). Asimismo, el concepto de garantía podría analogarse también a lo que Pollock llamó, en el ámbito de la inteligencia artificial, un esquema de razonamiento o esquema de razón (Pollock y Gillies, 2000, p. 243). Esto quiere decir que no se trata de los fundamentos de nuestras argumentaciones, se trata en verdad de un tipo de reglas particulares (reglas sustantivas, no formales) que descubrimos en la praxis argumentativa, y nos sirven para extraer conclusiones a partir de un conjunto de razones.

La estrecha relación entre la lógica derrotable y los esquemas de razonamiento de Pollock y por extensión a los esquemas de argumentación, en la comprensión que de ellos tiene Walton, Reed y Macagno (2008), abre la posibilidad de convergencia entre los desarrollos técnicos de inteligencia artificial para representar el razonamiento de sentido común y los modelos analíticos de la argumentación, como es el caso del modelo de Toulmin.

La formalización del razonamiento no monótono o derrotable como subcategoría, supone lidiar con algún tipo de inconsistencias, como es el caso en los conflictos de deberes prima facie, y requiere también alguna estrategia computablemente eficiente para manejarlas sin caer en una trivialización de 
un sistema que modelice el uso de argumentos derrotables. Estas exigencias teóricas se tornaron operativas con la propuesta de Reiter (1980).

A modo de cierre de esta parte, es relevante señalar que la argumentación se ha venido utilizando desde hace al menos un par de décadas para estudiar el razonamiento derrotable (Chesñevar, Maguitman y Loui, 2000) $\mathrm{y}$, recientemente, se han desarrollado marcos de argumentación abstracta (Prakken y Vreeswijk, 2002) para apoyar la caracterización de razonamiento no-monótono en términos de teoría de la argumentación. Los elementos básicos de estos marcos de referencia son las nociones de argumento y aceptabilidad de un argumento, apropósito de criterios de garantía. En pocas palabras, un argumento es aceptable solo si se muestra que no es posible de refutar con argumentos más fuertes.

\section{Esquemas argumentativos}

¿Qué circunstancias, informaciones o eventos debilitan a los argumentos? Es una pregunta invariable que sólo se puede responder utilizando los criterios que, de acuerdo con Toulmin, varían de un campo a otro campo argumentativo.

Esta simple respuesta e idea se deja apreciar con facilidad en el desarrollo que, especialmente, Walton (Walton y Krabbe, 1995; Walton, 1996a, 1996b, 1997, 2004, 2007; Walton, Reed, Macagno, 2008) ha entregado a largo de los últimos años, y que se captura en la noción de esquema argumentativo y preguntas críticas asociadas a cada esquema identificado.

Un ejemplo arroja luz de inmediato para entender lo implicado y el vínculo con Toulmin. Supongamos que frente a un caso criminal complejo el juez recurre al experto u opinión del perito. El juez no sólo recurre a esta posibilidad en tanto oportunidad procedimental del sistema legal, sino que accede a ella en virtud de un razonamiento y/o patrón intuitivo sobre el que descansa su práctica profesional, pero también su razonamiento práctico: esquema argumentativo de apelación al experto. Ciertamente, el defensor puede dudar de que el experto en cuestión sea tal o tenga ese estatus, incluso puede debilitar su testimonio aduciendo incompatibilidad, incongruencias y contradicciones de tal experto con lo que ha hecho en el pasado, ha declarado en otros casos, o porque ha señalado algo que otros expertos en el mismo dominio no sostienen.

La apelación al experto se reproduce modélicamente de la siguiente forma:

Esquema: 
Premisa mayor: La fuente $\mathrm{N}$ es un experto en el tema del dominio $\mathrm{D}$ que contiene proposición A.

Premisa menor: $\mathrm{N}$ sostiene que la proposición A (en el dominio D) es verdad (falsa)

Conclusión: A puede plausiblemente ser tomada como verdad (falso)

Las preguntas críticas aparecen allí donde el agente epistémico que lo utiliza se enfrenta a un par epistémico que, en un diálogo, razona con las mismas posibilidades que tal esquema le otorga, esto es, a partir del espacio de revisión que contiene el dominio del experto una vez se usa en deliberación. En nuestro ejemplo, entre otras preguntas que se pueden plantear, se encuentran las siguientes:

1. Pregunta por lo experto: ¿Qué tan creíble es N como fuente experta?

2. Pregunta por el campo: ¿Es $\mathrm{N}$ un experto en el campo en el que A está?

3. Pregunta por la opinión: ¿Qué dijo N que implica A?

4. Pregunta por la confiabilidad: ¿Es N confiable como una fuente?

5. Pregunta por la consistencia: ¿Es A consistente con lo que otros expertos sostienen?

6. Pregunta por la evidencia: ¿Lo que sostiene $\mathrm{N}$ está basado en evidencia?

$\mathrm{Si}$ alguna de estas preguntas se responde negativamente, y contrariamente al flujo presuntivo del contenido proposicional del esquema, entonces emerge la posibilidad de derrota en su uso. Además, como se observa, estas preguntas son absolutamente propias del dominio o campo en el que se suscribe el esquema argumentativo. De acuerdo con Walton, Reed y Macagno (2008), los esquemas argumentativos son formas de argumentos en tanto patrones estereotipados de razonamiento humano, y como tal son derrotables y presuntivos. Es en el acto de reconstrucción de la forma completa del esquema en que agente proponente y agente recipiente recuperan todas las premisas implícitas de tal cadena, siendo su derrotabilidad natural adherida la que puede extender con cierta holgura los vínculos inferenciales.

Respecto de la ligazón entre campo argumentativo y esquema argumentativo (y las preguntas críticas asociadas a un esquema argumentativo), en la década de 1950 Toulmin (2007) ya nos ofrecía una definición de la noción de campo que contenía en ciernes los desarrollos producidos posteriormente por la lógica informal. Para Toulmin los campos argumentativos son cierto(s) dominio(s) temático(s), o disciplinas, en los que se utilizan ciertas reglas de pasaje o principios reguladores para la construcción de argumentos. Vincula estrechamente la noción de campo con la noción, por un lado, de tipo lógico, y con los términos fuerza y criterio. En la primera relación establece: 
For the sake of brevity, it will be convenient to introduce a technical term: let us accordingly talk of a field of argument. Two arguments will be said to belong to the same field when the data and conclusions in each of the two arguments are, respectively, of the same logical type: they will be said to come from different fields when the backing or the conclusions in each of the two arguments are not the same logical type. (1958, p. 14)

La triangulación entre campo, fuerza y criterio, se observa en que la fuerza de los términos modales (posible, imposible, necesario, innecesario, etc.) es independiente del campo, pues cumple la misma función en cada argumento, pero los criterios, en tanto razones o bases por los que son aplicados estos términos modales en determinado contexto, son dependientes de los campos, ya que a partir de éstos se puede determinar si los primeros han sido ocupados correctamente. En este contexto, las prácticas epistemológicas de cada campo o dominio organizarían los parámetros dialécticos que establecen los tipos de preguntas críticas pertinentes. Dicho de otra forma, los criterios para evaluar argumentos serían internos respecto de los dominios, disciplinas y prácticas situadas.

Nótese que el esquema básico de Toulmin también contiene esta derrotabilidad natural que todo esquema argumentativo comparte. El modelo tiene inserto una dialéctica que funciona con, y representa a, una derrotabilidad recursiva: si la pretensión es desafiada (¿sobre qué razones aseveras x?, se aducen razones (datos); si el paso de los datos a la pretensión es desafiado (¿cómo justificas pasar de esos datos a tal pretensión?), entonces se aduce una regla de inferencia (garantía); si la garantía es desafiada (¿es realmente un paso seguro?), se aduce el apoyo; si se desafía la fuerza de la pretensión (¿qué tan fuerte es tu pretensión?), se aducen cualificadores; y si se pregunta por debilitadores al argumento (¿qué podría obstaculizar tu argumento?), se señalan las condiciones de refutación. Este proceso lo reconstruye cada uno de nosotros, agentes epistémicos, en la vida cotidiana sin otro talento que el sentido común.

\section{Conclusiones}

Coincidiendo con Paglieri y Castelfranchi (2005), es posible afirmar que el modelo de análisis de Toulmin es un marco de referencia para entender cómo funciona la derrotabilidad de los argumentos de sentido común. El diseño analítico de Toulmin no solamente permite establecer una analogía general entre el matizador de la conclusión (Q) y la representación de una inferencia 
derrotable, sino que sobre todo tiene un valor heurístico esencial para introducir el estudio del razonamiento práctico en contextos interdisciplinarios.

Siguiendo a Prakken (2005, p. 12), hay al menos tres lecciones fundamentales que se pueden obtener de Toulmin: en primer lugar, que las premisas de un argumento pueden tener diferentes papeles, es decir, diferentes esquemas de argumento con roles estereotípicos de premisas distintos; en segundo lugar, que los argumentos cotidianos y en general todos los esquemas de razonamiento práctico son derrotables, lo que Toulmin captó en su noción de rebuttal; y tercero, que los estándares para la evaluación de argumentos son dependientes de campo. Esto es, dependientes de diferentes respaldos con garantías específicas y distintivas.

Este punto cobra relevancia por cuanto los estándares pueden ser definidos por medio de la identificación de esquemas empleados en un determinado campo y formulando los debilitadores (undercutting defeaters) que corresponden a las cuestiones críticas de estos esquemas. Ya que diferentes campos pueden tener sus propios esquemas argumentativos típicos, los criterios para la evaluación de los argumentos diferirán para cada campo.

Aunque, por ejemplo, en el trabajo de la inteligencia artificial y el derecho se ha sustituido el esquema argumentativo original de Toulmin con un análisis más refinado, lo han hecho dentro del espíritu del desafío de Toulmin, que fue desarrollar una explicación de la validez del razonamiento aplicado a la argumentación cotidiana. Un hecho trascendente es que la inteligencia artificial y el derecho han demostrado que dicha explicación representacional todavía puede ser formal y computacional. Toulmin, de hecho, contra lo que a veces erróneamente se ha difundido, no quiso abolir ni erradicar la lógica clásica, sólo debilitó su predominio en la evaluación de los procesos argumentativos y con ello la situó, con el tiempo, en su justo lugar.

\section{Referencias}

ARISTOTE. "Topiques". Tome I, Livres I-IV. Texte établi et traduit par Jacques Brunschwig. Paris: C. U, 1967.

ALCHOURRÓN, C. "Philosophical Foundations of Deontic Logic and the Logic of Defeasible Conditionals". In: J. J. Meyer, R. J. Wieringa (ed.). Deontic Logics in Computer Science: Normative System Specification (pp. 43-84). Cichester: Wiley \& Sons, 1993.

BILlingTON, D. "Defeasible Logic is Stable". Journal of Logic and Computation, 3, pp. 370-400, 1993. 
CARNOTA, R. J. "Lógica e Inteligencia Artificial". In: C. Alchourrón, J. Méndez, R. Orayen. Enciclopdia Iberoamericana de Filosofía. Lógica (pp. 144-183). Madrid: Editorial Trotta. 2005.

CHESÑEVAR, C., MAGUITMAN, A., LOUI, R. "Logical Models of Argument". ACM Computing Surveys, Nr. 32, Vol. 4, p. 337-383, 2000.

CHISHOLM, R. "Perceiving: A Philosophical Study". Ithaca: Cornell University Press, 1977.

EVANS, J. "Introducción a los Tópicos de Aristóteles". Buenos Aires: Ciudad Argentina, 1999.

GABBAY, D., WOODS, J. et al. "Handbook of the logic Argument and Inference (The Turn Towards the Practical)". Vol. 1. London: North Holland Published, 2002.

GOLDMAN, A. "What is Justified Belief?". In: G. Pappas (ed.). Justification and Knowledge: New Studies in Epistemology (pp. 1-23). Dordrecht: Reidel, 1979.

GOVIER, T. "Problems in Argument Analysis and Evaluation". Amsterdam: Mouton de Gruyter/Foris, 1987.

HANSSON, B. "An Analysis of Some Deontic Logics”. Noûs, 3, pp. 373-398, 1969.

HARMAN, G. "The Inference to the Best Explanation". The Philosophical Review, Nr. 74, Vol. 1, pp. 88-95, 1965.

HART, H. "The ascription of responsibility and rights". Proceedings of the Aristotelian Society (1948-9), pp. 171-194, 1949.

HORTY, J. “Reasons as Defaults". Philosophers' Imprint, Nr. 3, Vol. 7, pp. 1-28, 2007.

LOUI, R. P. "Hart's critics on defeasible concepts and ascriptivism" In: Proceedings of the Fifth International Conference on Artificial Intelligence and Law (pp. 21-30). New York: ACM Press, 1995.

MAHER, M. "Propositional Defeasible Logic Has Linear Complexity". Theory and Practice of Logic Programming, Nr. 1, Vol. 6, pp. 691-711, 2001.

MAHER, M. J. et al. "Efficient Defeasible Reasoning Systems". International Journal on Artificial Intelligence Tools, Nr. 10, Vol. 4, pp. 483-501, 2001.

NUTE. D. "Defeasible Logic". In: D. Gabbay, C. Hogger, J. Robinson (ed.). Handbook of Logic in Artificial Intelligence and Logic Programming. Vol. 3 (pp. 353-395). New York: Oxford University Press, 1994.

PAGLIERI, F., CASTELFRANCHI, C. "Arguments as belief structures: Towards a Toulmin layout of doxastic dynamics?". In: D. Hitchcock (ed.). The uses of argument (pp. 356-367). Hamilton: OSSA, 2005.

PEIRCE, C. S. "What is a leading principle?”. In: J. Buchler (ed.). Philosophical Writings of Peirce (pp. 129-134). New York: Dover, 1955.

POLLOCK, J. "Criteria and our knowledge of the material world". The Philosophical Review, Nr. 76, Vol. 1, pp. 28-60, 1967.

1974.

"Knowledge and Justification". New Jersey: Princeton University Press, . "Defeasible reasoning". Cognitive Science, Nr. 11, Vol. 4, pp. 481-518, 1987. 
1995.

. "Practical Reasoning in Oscar". Philosophical Perspectives, 9, pp. 15-48,

. "Contemporary Theories of Knowledge". With J. Cruz, second edition. Lanham: Rowman-Littlefield, 1999.

. "Defeasible reasoning with variable degrees of justification". Artificial intelligence, 133, pp. 233-282, 2001.

POLLOCK, J., GILLIES, A. "Belief revision and epistemology". Synthese, 122, pp. 69-92, 2000.

PRAKKEN, H. “AI \& Law, Logic and Argument Schemes”. Argumentation, 19, pp. 303-320, 2005.

PRAKKEN, H., VREESWIJK, G. "Logical Systems for Defeasible Argumentation". In: D. Gabbayy, F. Guenther (ed.). Handbook of Philosophical Logic. Vol. 4 (pp. 219318). Dordrecht: Kluwer, 2002.

PRAKKEN, H., REED, C., WALTON, D. “Argumentation schemes and generalisations in reasoning about evidence". In: Proceedings of the Ninth International Conference of Artificial Intelligence and Law (pp. 32-41). New York: ACM Press, 2003.

REITER, R. "A logic for default reasoning”. Artificial Intelligence, 13, pp. 81-132, 1980.

ROSS, W. "The Right and the Good". Oxford: Clarendon Press, 1930.

TOULMIN, S. "The Uses of Argument". Cambridge: Cambridge University Press, 1958.

. "Los usos de la argumentación”. Barcelona: Península, 2007.

TOULMIN, S., RIEKE, R., JANIK. A. "An Introduction to Reasoning”. New York: Macmillan Publishing Co., Inc., 1979.

VELASCO, I. "No-monotonicidad: un nuevo paradigma inferencial”. In: J. Martínez, y A. Ponce de León (coord.). El saber filosófico. Vol. 2 (pp. 361-376). México: Editorial Siglo XXI, 2007.

WALTON, D. “Informal Logic”. New York: Cambridge University Press, 1989. . "Argumentation schemes for presumptive reasoning". Mahwah: LEA, 1996a. . "Argument Structure: A Pragmatic Theory". Toronto: University of Toronto Press, $1996 \mathrm{~b}$.

. “Appeal to Expert Opinion”. Pennsylvania: Pennsylvania State University Press, 1997.

. "Abductive reasoning". Tuscaloosa: The University of Alabama Press, 2004. "Media Argumentation. Dialectic, Persuasion, and Rhetoric". Cambridge: Cambridge University Press, 2007.

WALTON, D., KRABBE, E. "Commitment in Dialogue. Basic Concepts of Interpersonal Reasoning”. New York: SUNY, 1995.

WALTON, D., REED, C., MACAGNO, F. "Argumentation Schemes". New York: Cambridge University Press, 2008. 